\title{
Effects of Amiodarone in a Swine Model of Nortryptiline Toxicity
}

\author{
Fermin Barrueto, Jr., MD a, Indira Murr, MD b Andrew Meltzer, MD a, \\ Kori Brewer, $P h D^{\mathrm{b}}$, and William Meggs, $M D, P h D^{\mathrm{b}}$
}

a University of Maryland School of Medicine, Baltimore, MD

b Brody School of Medicine at East Carolina University, Greenville, NC

\begin{abstract}
Objectives: Antiarrhythmics can have devastating effects in cardiotoxic poisonings. Amiodarone is recommended for treatment of wide complex tachycardia, but its hemodynamic effects in wide complex tachycardia induced by tricyclic antidepressant poisoning are unknown. The objective of this study was to compare the effects of sodium bicarbonate, amiodarone, and normal saline in treating wide complex tachycardia secondary to nortriptyline poisoning.

Methods: This unblended randomized controlled animal study involved 18 anesthetized, intubated pigs with arterial and venous lines. Nortriptyline $(2 \mathrm{mg} / \mathrm{mL})$ was infused at $20 \mathrm{mg} / \mathrm{min}$ until the onset of toxicity, defined as a systolic blood pressure $\leq 50 \mathrm{mmHg}$ or $\mathrm{QRS} \geq 120 \mathrm{~ms}$. At that point, the pigs were randomized into three groups of six. Group I received $0.9 \% \mathrm{normal}$ saline, $10 \mathrm{ml} / \mathrm{kg}$. Group II received hypertonic sodium bicarbonate, $1 \mathrm{mEq} / \mathrm{kg}$. Group III received amiodarone, $15 \mathrm{mg} / \mathrm{kg}$. The pigs were observed until death or survival at 60 minutes.

Results: After treatment, the changes in QRS were as follows: Group I, $-2.0 \mathrm{~ms}$; Group II, -33.0 ms; Group III, -21.7 ms. ANOVA demonstrated no significant difference between the groups $(\mathrm{p}=0.28)$. Mean arterial pressures 10 minutes after treatment were as follows: Group I, $19.4 \mathrm{mmHg}$; Group II, $23.7 \mathrm{mmHg}$; Group III, $12.5 \mathrm{mmHg}$. Based on ANOVA, there was no significant difference between any of the groups $(\mathrm{p}=0.50)$.

Conclusions: In this model of nortriptyline poisoning, the administration of amiodarone to correct wide complex tachycardia did not have a harmful effect.
\end{abstract}

\section{INTRODUCTION}

Some medications have the potential to cause devastating effects when administered in the setting of drug overdoses. Examples include treatment of digoxin toxicity and resultant hyperkalemia with calcium, treatment of cocaine toxicity with beta blockers, and treatment of tricyclic antidepressant overdoses with physostigmine [1-3]. The hemodynamic effects of amiodarone in the treatment of wide complex tachycardias are unknown when the cause is a tricyclic antidepressant overdose.

Keywords: amiodarone, nortryptiline, tachycardia, poisoning, tricyclic antidepressants

Acknowledgements: The authors thank Linda J. Kesselring, MS, ELS, technical editor/writer in the Department of Emergency Medicine at the University of Maryland School of Medicine, for her invaluable assistance with the preparation of this manuscript.

Notes: Presented at the Residents Research Forum, Division of Emergency Medicine, University of Maryland School of Medicine, Baltimore, Maryland, June 1, 2005, and at the Scientific Assembly of the American College of Emergency Physicians, Washington, DC, September 2005.

This study was supported by the East Carolina University Resident Research Fund.

Corresponding Author: Fermin Barrueto, Jr., MD, Division of Emergency Medicine, University of Maryland School of Medicine, 110 South Paca Street-6th floor, Suite 200, Baltimore, MD 21201. Email: fbarr001@umaryland.edu 
The Advanced Cardiac Life Support (ACLS) protocol for the treatment of wide-complex tachycardia of unknown etiology calls for the administration of amiodarone [4-5]. Although this approach is appropriate for patients with ischemic heart disease, ACLS might exacerbate cardiac dysfunction in a toxin-induced wide-complex tachycardia such as that caused by an overdose of a tricyclic antidepressant (TCA), a sodium channel blocker. Sodium bicarbonate is known to effectively reverse TCA cardiotoxicity. In some TCA poisonings, the etiology may be unknown, and amiodarone may be inadvertently administered. Hence, it is important to know if amiodarone causes an adverse effect in TCA overdoses.

An initial mouse survival study showed that there was no statistical difference in the amitriptyline $\mathrm{LD}_{50}$ of mice pretreated with amiodarone when compared to a normal saline control [6]. Since hemodynamic monitoring and electrocardiographic (ECG) analysis were not possible in that small-animal model, a swine model was devised to evaluate these critical measures.

Using a swine model of severe nortriptyline poisoning developed by McCabe et al, the purpose of this study was to determine if amiodarone, a class III antidysrrhythmic, had harmful hemodynamic effects on a wide-complex tachycardia [7]. The null hypothesis is that there would be no difference in the QRS complex or mean arterial pressure between TCA-poisoned subjects treated with amiodarone and those treated with hypertonic sodium bicarbonate, the standard of care for known TCA poisoning.

\section{MATERIALS AND METHODS}

The Animal Care and Use Committees of the participating universities approved this experiment. This was a non-blinded, randomized controlled animal study evaluating the effects of amiodarone in a swine model of severe nortriptyline toxicity.

A change of $50 \%$ in QRS duration was considered significant in sample size calculations made with ANOVA. Ten pigs were found to have a $90 \%$ chance of finding a $50 \%$ difference for a single endpoint of a QRS duration at a fixed time. This is comparing one treatment modality to control; for example, amiodarone versus control (normal saline) or hypertonic sodium bicarbonate versus control (normal saline). Subjects were 18 male domestic swine weighing 10 to 13 kilograms. All animals were prepared and instrumented in the same fashion and randomly assigned to three treatment arms. This model was adapted from that described by McCabe et al [7].

The dosing of each arm was determined by information from previous literature; the only exception was the normal saline dose because it was the negative control arm. We wanted to administer a volume that was similar to the amiodarone and sodium bicarbonate arms to determine the toxic effects of this nortriptyline infusion. Amiodarone has been found to increase the ventricular fibrillatory threshold in a dose-dependent fashion [8]. Utilizing a maximal and safe dose found in a previous swine model, $15 \mathrm{mg} / \mathrm{kg}$ of amiodarone would clinically parallel an administered $300 \mathrm{mg}$ bolus [8]. The clinical dose of sodium bicar- bonate is $1-2 \mathrm{mEq} / \mathrm{kg}$, and thus we administered the first bolus, $1 \mathrm{mEq} / \mathrm{kg}$, to our swine model. This would also assist with keeping the volume infused equivalent in all arms.

The animals were intramuscularly sedated with a mixture of $1 \mathrm{mg} / \mathrm{kg}$ of xylazine and tiletamine hydrochloride (Telazol) (5 mg/mL). An ear vein was cannulated, and each animal was intubated with a 5.0 endotracheal tube and maintained on $1 \mathrm{mg} / \mathrm{mL}$ of inhaled isofluorane. ECG leads were placed, and lead II was continuously monitored. A femoral arterial line was placed either percutaneously or via femoral cutdown for continuous monitoring of blood pressure and blood sampling. The pig was allowed 5 minutes after instrumentation and anesthesia to acquire baseline parameters. Nortriptyline (Sigma-Aldridge Chemical, St. Louis) was added to normal saline to a final concentration of $2 \mathrm{mg} / \mathrm{mL}$ and intravenously infused at a rate of $20 \mathrm{mg} / \mathrm{min}$ until toxicity was reached. Toxicity was defined as a systolic blood pressure $\leq 50 \mathrm{mmHg}$ or a QRS duration $\geq 120 \mathrm{~ms}$. After toxicity was achieved, a continuous infusion of the nortriptyline solution was maintained at a rate of $5 \mathrm{mg} / \mathrm{min}$. At this point, group I, serving as a negative control, received $10 \mathrm{ml} / \mathrm{kg}$ of $0.9 \%$ normal saline solution; group II, serving as a positive control, received $10 \mathrm{ml} / \mathrm{kg}$ of an $8.4 \%$ sodium bicarbonate solution; and group III received $15 \mathrm{mg} / \mathrm{kg}$ of amiodarone solution followed by normal saline to equal a total volume of $10 \mathrm{ml} / \mathrm{kg}$. In one case, one of the pigs from group I was removed from the study because of a failure of the infusion pump; therefore, although 18 animals were used for the study, only data from 17 pigs could be statistically analyzed.

All animals were observed for 60 minutes after the onset of toxicity or until death. Death was defined as asystole or zero blood pressure for at least 60 seconds. Surviving animals were intravenously euthanized with sodium pentobarbital $(100 \mathrm{mg} / \mathrm{kg})$. Blood pressure, pulse, and QRS duration were measured at 5minute intervals. Arterial $\mathrm{pH}, \mathrm{PCO}_{2}$, and $\mathrm{PO}_{2}$ were measured at baseline and every 15 minutes thereafter. Serum nortriptyline levels were measured at toxicity. The outcome measures, posttreatment change in QRS duration, and mean arterial blood pressure (MAP) 10 minutes after treatment were compared between groups using ANOVA.

\section{RESULTS}

Prior to the administration of intravenous nortriptyline, baseline parameters of weight $(\mathrm{kg}), \mathrm{pH}, \mathrm{QRS}$ duration, mean arterial pressure, all pulse and nortriptyline serum concentration were similar across groups (Table 1).

Wide complex tachycardia, defined as a QRS duration $\geq 120 \mathrm{~ms}$, occurred in 16 of the 17 pigs. All 5 animals in the negative control group I (NS) developed wide complex tachycardia and deteriorated to asystole. All 6 animals in the positive control group II $\left(\mathrm{NaHCO}_{3}\right)$ experienced wide complex tachycardia; 3 of the animals deteriorated to asystole. Five of the 6 animals in group III (amiodarone) experienced wide complex tachycardia; 4 of them deteriorated to asystole. (See Figure 1 for time of 
TABLE 1. Baseline Parameters

\begin{tabular}{lccc}
\hline $\begin{array}{l}\text { Mean Parameters } \\
\text { (Baseline) }\end{array}$ & $\begin{array}{c}\text { Normal Saline } \\
(\mathbf{N}=\mathbf{5})\end{array}$ & $\begin{array}{c}\mathbf{N a H C O}_{\mathbf{3}} \\
\mathbf{( N = 6 )}\end{array}$ & $\begin{array}{c}\text { Amiodarone } \\
(\mathbf{N}=\mathbf{6})\end{array}$ \\
\hline Weight $(\mathrm{kg})$ & $11.02 \pm 2.24$ & $11.51 \pm 1.61$ & $11.07 \pm 0.87$ \\
\hline $\mathrm{pH}$ & $7.48 \pm 0.02$ & $7.44 \pm 0.02$ & $7.46 \pm 0.06$ \\
\hline $\mathrm{QRS}(\mathrm{msec})$ & $76.0 \pm 8.9$ & $80.0 \pm 22.8$ & $63.3 \pm 10.3$ \\
\hline MAP & $71.2 \pm 14.6$ & $73.0 \pm 12.7$ & $62.2 \pm 7.1$ \\
\hline Pulse (beats/min) & $129 \pm 19$ & $128 \pm 22$ & $127 \pm 21$ \\
\hline$[\mathrm{TCA}]$ level (ng/L) & $10500 \pm 4266$ & $10035 \pm 6817$ & $7708 \pm 2860$ \\
\hline
\end{tabular}

death). The mean changes in QRS at 10 minutes after treatment were as follows: saline, narrowed by $2.0 \mathrm{~ms}$; bicarbonate, narrowed by $33.0 \mathrm{~ms}$; amiodarone, narrowed by $21.7 \mathrm{~ms}$. There was no statistically significant difference between the groups after treatment $(\mathrm{p}=0.28)$, though the trend favored sodium bicarbonate (Figure 2).

MAP at 10 minutes after treatment was measured in negative control group I (NS), positive control group II $\left(\mathrm{NaHCO}_{3}\right)$, and in test group III (amiodarone). In the negative control group I (NS), the MAP was $19.4 \mathrm{mmHg}$; in the positive control group II ( $\left.\mathrm{NaHCO}_{3}\right)$, MAP was $23.7 \mathrm{mmHg}$; and in test group III (amiodarone), MAP was $12.5 \mathrm{mmHg}$. There was no statistically significant difference between the groups $(p=0.50)$. Again, the trend indicated that sodium bicarbonate was superior (Figure 3).
Survival rates at 60 minutes were as follows: Group I: 0/5(0\%), Group II: 3/6 (50\%), and Group III 2/6(33\%) (Figure 1). After analysis of our primary outcome measure, change in QRS, our power was $<50 \%$; 169 animals per group would be required to demonstrate $95 \%$ power. Analysis of the secondary outcome measure of MAP demonstrated a power of $50 \%$ to $80 \%$. To obtain 95\% power, 17 animals per group would be needed.

\section{DISCUSSION}

In this model of nortriptyline poisoning, administering amiodarone to treat wide complex tachycardias was not harmful. Hypertonic sodium bicarbonate actually showed the most favorable trend with regard to MAP and QRS duration and was superior to amiodarone. Interestingly, it has been assumed that the narrowing of the QRS indicates improvement of inotropy; however, in the amiodarone group, the three swine that had a narrowing of the QRS complex died.

The sodium channel blockade with resultant negative inotropy in combination with the $\alpha$-blocking effects of TCAs places a patient in a precarious hemodynamic state; theoretically, amiodarone could exacerbate that state. Although this swine study did not demonstrate this additive effect to the point of statistical significance, the possibility may be likened to the theoretic concern about administering calcium to a digoxin-poisoned patient and causing "stone heart". [9] Since amiodarone is known to cause hypotension and QT prolongation, a potential physiologic mechanism exists.

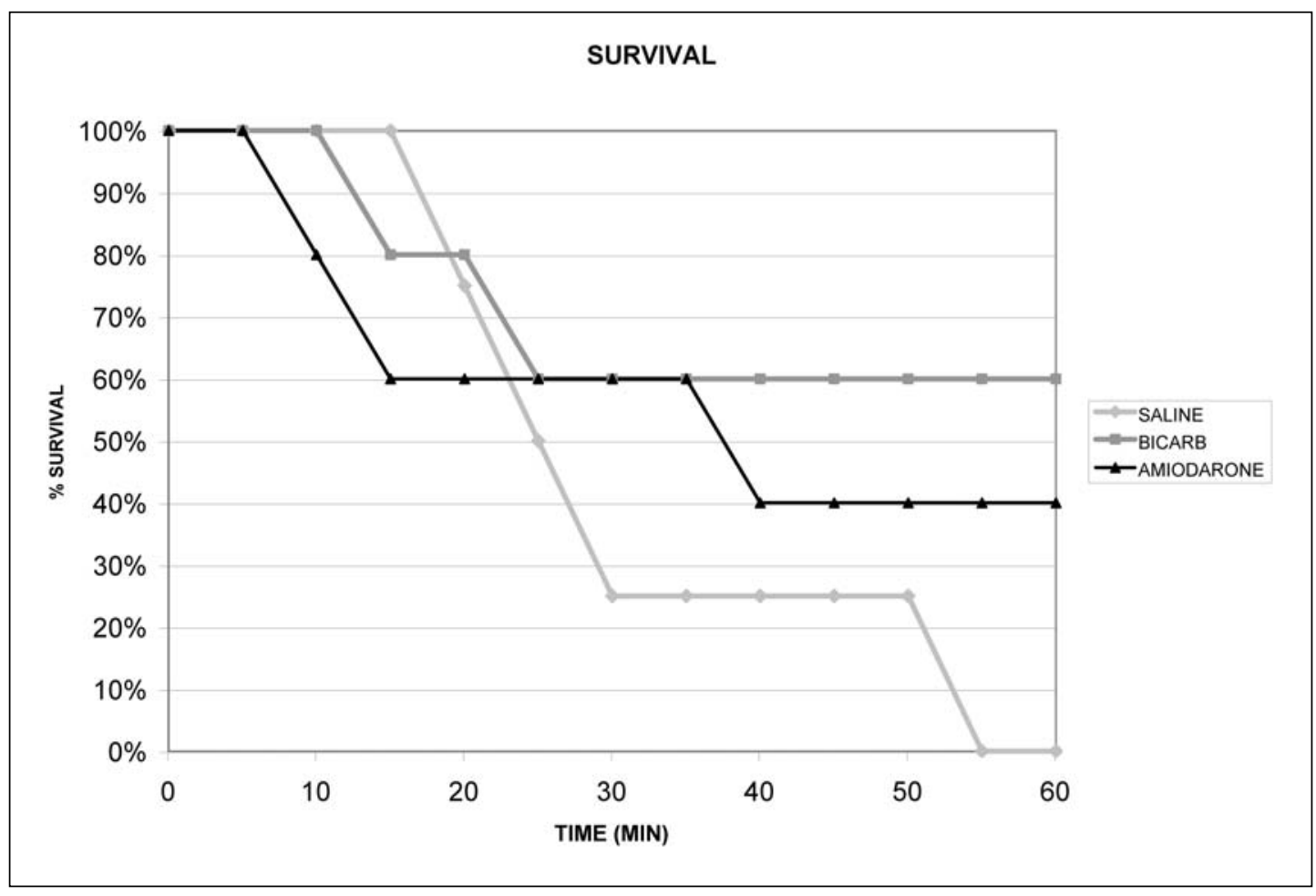

Figure 1. Time to death for each animal. 


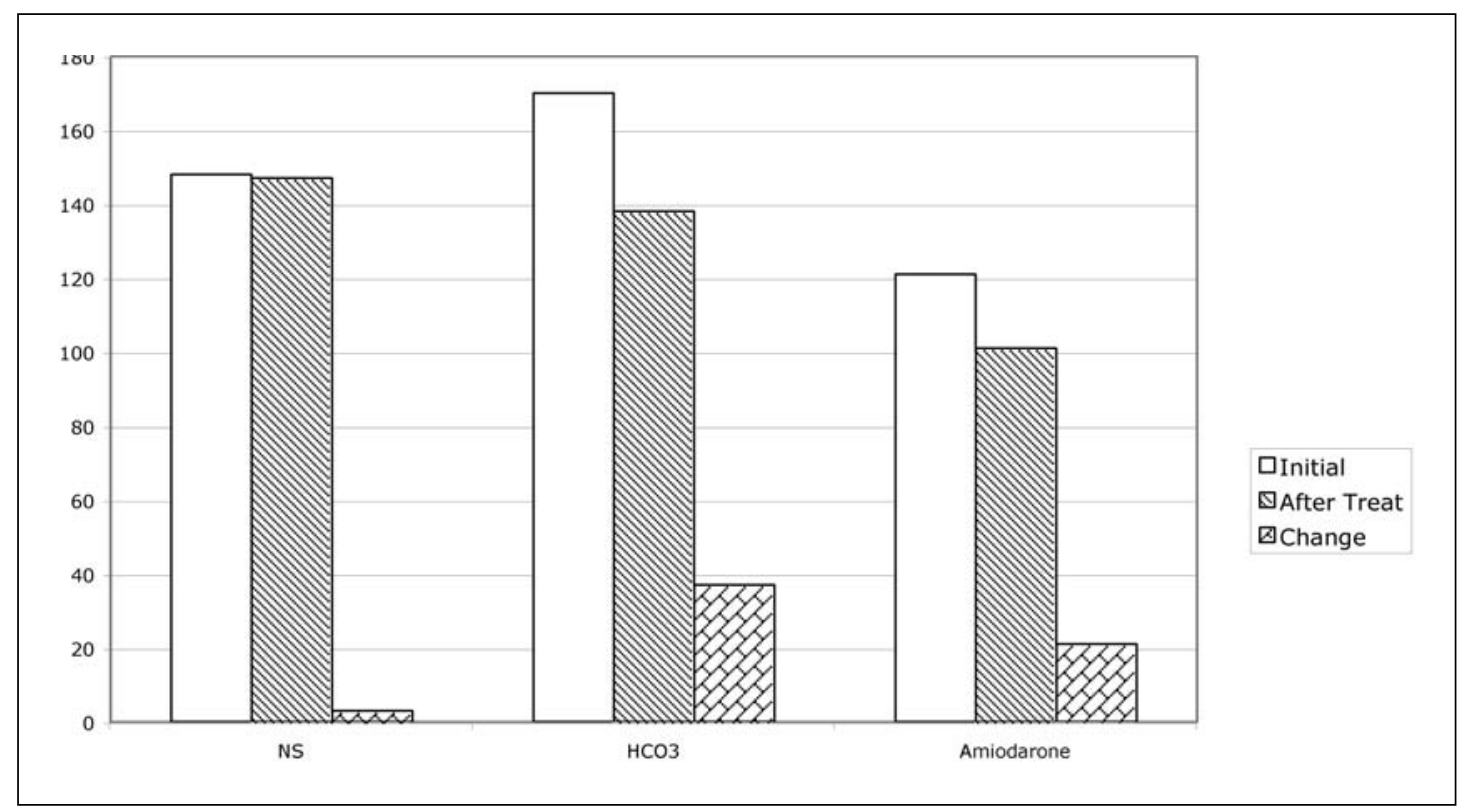

Figure 2. QRS at initial toxicity and at 10 minutes after treatment; change in QRS.

Amiodarone did not appear to have a harmful effect in this limited animal model of severe nortriptiline toxicity. Further research is required to determine if amiodarone is beneficial in other toxin-induced wide complex tachycardias. Another arm that combines amiodarone and sodium bicarbonate would have been interesting and may be a future direction for this work.

\section{LIMITATIONS}

The animal model does not reproduce human toxicity though the swine model does provide a close approximation of the human cardiovascular system. The route of exposure that is typically seen in a clinical setting is oral and not intravenous as was

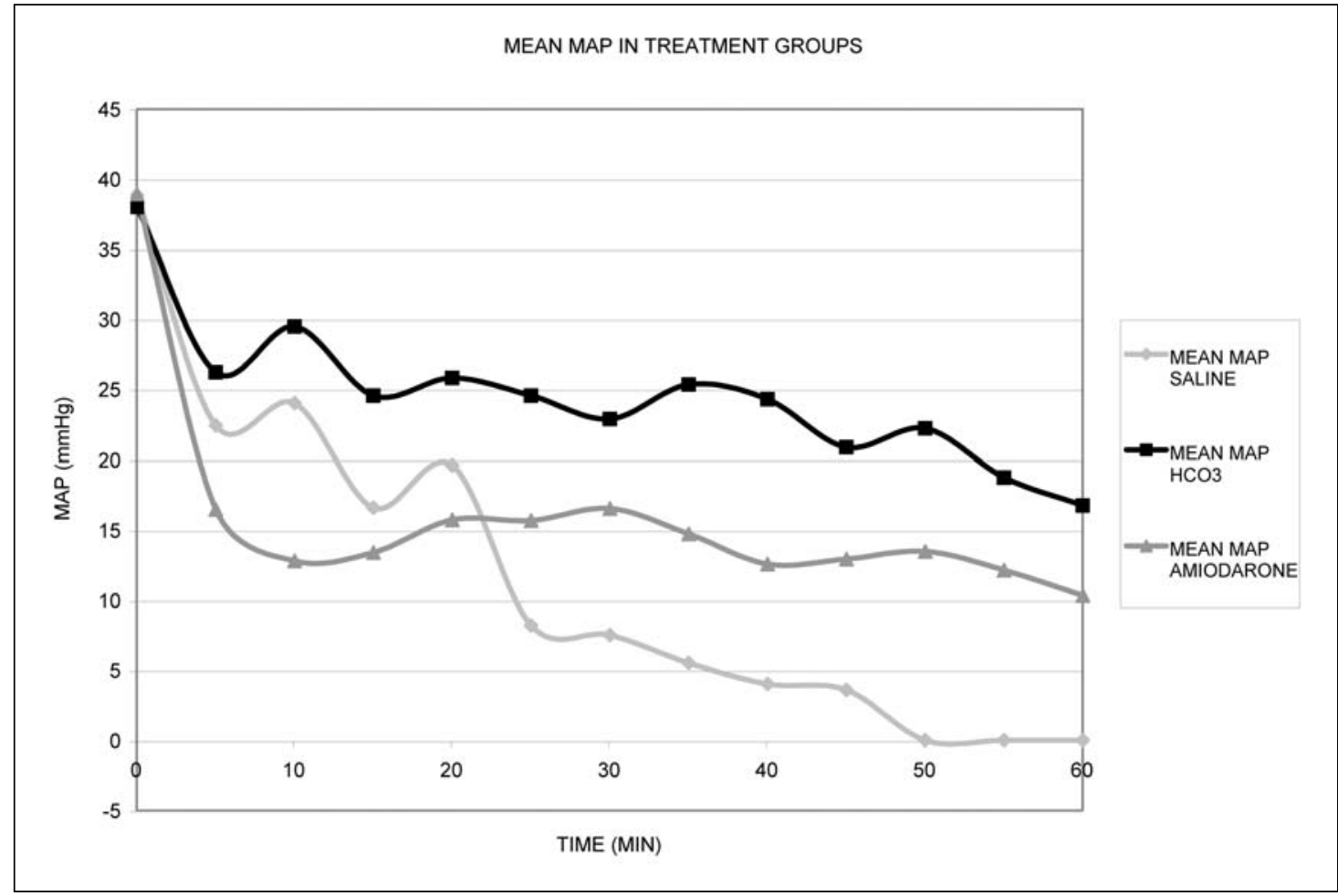

Figure 3. Mean MAPs for each arm. 
performed in this study. In this study, a single dose of sodium bicarbonate was given; but in clinical practice, an initial bolus is followed by an infusion. The efficacy of sodium bicarbonate relative to amiodarone could have been enhanced if larger doses and infusions of sodium bicarbonate had been instituted. In this study, more swine would have improved our statistical power. Analysis showed that 17 more animals per group would be needed, but that number is not practical to manage in a laboratory setting. The investigators were not blinded to treatment and would have added scientific validity. Also, another amiodarone arm administered without TCAs would allow us to compare its typical effect on MAP and QRS duration with the effect we have seen in a model of severe nortryptiline poisoning.

The authors have no potential conflicts of interest to report.

\section{REFERENCES}

1. Van Deusen SK, Birkhahn RH, Gaeta TJ. Treatment of hyperkalemia in a patient with unrecognized digitalis toxicity. J Toxicol Clin Toxicol 2003;41(4):373-376.

2. Lange RA, Cigarroa RG, Flores ED, McBride W, Kim AS, Wells PJ, et al. Potentiation of cocaine-induced coronary vasoconstriction by beta-adrenergic blockade. Ann Intern Med

1990;112(12):897-903.
3. Pentel P, Peterson CD. Asystole complicating physostigmine treatment of tricyclic antidepressant overdose. Ann Emerg Med 1980;9(11):588-590.

4. Caron MF, Kluger J; White CM. Amiodarone in the new AHA guidelines for ventricular tachyarrhythmias. Ann Pharmacother 2001;35:1248-1254.

5. Kudenchuk PJ, Cobb LA, Copass MK, Cummins RO, Doherty AM, Fahrenbruch CE, et al. Amiodarone for resuscitation after out-of-hospital cardiac arrest due to ventricular fibrillation. N Engl J Med 1999;341:871-878.

6. Barrueto F Jr, Chuang A, Cotter BW, Hoffman RS, Nelson LS. Amiodarone fails to improve survival in amitriptylinepoisoned mice. Clin Toxicol 2005;43(3):147-149.

7. McCabe JL, Cobaugh DJ, Menegazzi JJ, Fata J. Experimental tricyclic antidepressant toxicity: a randomized, controlled comparison of hypertonic saline solution, sodium bicarbonate and hyperventilation. Ann Emerg Med

1998;32:329-333.

8. Zhou L, White CM, Chen BP, Chow MS, Fan C, Kluger J. A comparison of the antifibrillatory effects of desethylamiodarone to amiodarone in a swine model. J Cardiovasc Pharmacol. 1999;34(3):440-5.

9. Hutchins GM, Silverman KJ. Pathology of the stone heart syndrome: massive myocardial contraction band necrosis and widely patent coronary arteries. Am J Pathol 1979;95(3):745-752. 